Gasarten zueinander liegen, kann bei Wasserstoff, Helium, Argon und Stickstoff verglichen werden. Die relative Lage der Minima bei diesen Gasarten stimmt zumindest qualitativ überein. In beiden Fällen zeigt der Stickstoff die Tendenz, bei vergleichsweise kleiner Magnetfeldstärke Minima zu durchlaufen. Die qualitative Abhängigkeit vom Gasdruck stimmt ebenso überein; die Lage des Minimums verschiebt sich schwächer als linear mit steigendem Druck zu höherer Magnetfeldstärke. Eine weitere Ähnlichkeit kann man darin sehen, daß in beiden Fällen die zugeführte Leistung von geringem Einfluß auf die Lage der Minima ist; dort ist die jeweils gewählte und dann konstant gehaltene Stromdichte, hier die Anodenspannung des Senders (im Druckbereich von 0,1 bis 0,6 Torr) ohne großen Einfluß. $\mathrm{Zu}$ stören scheint zunächst, daß bei den Hochfrequenzversuchen die Lage der Minima von der Senderfrequenz abhängt $\left(H_{\mathrm{m}} \sim V f\right)$. Diese Abhängigkeit könnte u. a. - durch den Einfluß der Senderfrequenz auf die Eindringtiefe hervorgerufen sein. Es ist gut denkbar, daß bei einer ringförmigen Hochfrequenzentladung die Eindringtiefe eine ähnliche Rolle spielt wie bei einer stati-

5 Mikrowellenmessungen von M. TUTTER an der hier betrachteten Hochfrequenzapparatur geben Anhalte dafür, daß nach dem Minimum Oszillationen auftreten. schen linearen Entladung der Gefäßradius $r$, für den bei Нон und Lehnert angenähert die Beziehung $H_{\mathrm{n}} \sim 1 / r$ beobachtet wurde. Für detailliertere Vergleiche der beiden Versuchsanordnungen und eine quantitative Prüfung der z. T. sicher komplizierten Zusammenhänge sind weitere theoretische und experimentelle Untersuchungen ${ }^{5}$, besonders der Temperaturverhältnisse und des Einflusses der Neutralgaskomponente, notwendig.

Bei den betrachteten Hochfrequenzentladungen ist die Leistungsdichte wesentlich höher als bei den Gleichstromentladungen von $\mathrm{HoH}$ und Lehnert. Außerdem ist hier ein relativ kurzes Entladungsgefä $\S^{6}$ mit außerhalb der Senderspule stark inhomogenem Magnetfeld benutzt, während dort sehr lange Entladungsrohre mit homogenem Magnetfeld verwendet wurden. Wie in diesem Falle kann auch bei früheren Hochfrequenzuntersuchungen, z. B. denen von Boeschoten, van INGEN und van DyK ${ }^{7}$ (kapazitive Ankoppelung), nur eine eingehende Prüfung entscheiden, ob und wie weit die anomale Diffusion für die beobachteten nicht-monotonen OHмschen Entladungswiderstände oder ähnliche Meßgrößen wesentlich ist.

${ }^{6}$ Auch bei G. MüLler wird ein kurzes Entladungsgefäß benutzt.

7 F. Boeschoten, A. M. van Ingen u. C. van Dyk, Interner Bericht, Utrecht.

\section{Massentransport in flüssigen Metallen senkrecht zu gekreuzten elektrischen und magnetischen Feldern}

\author{
Von Hans KnoF
}

Max-Planck-Institut für Chemie (Otto-Hahn-Institut), Mainz (Z. Naturforschg. 15 a, 745-746 [1960] ; eingegangen am 20. Juli 1960)

In flüssigem Quecksilber wandern gelöste Metalle unter dem Einfluß eines elektrischen Feldes. Mit theoretischen Überlegungen läßt sich für gekreuzte elektrische und magnetische Felder eine zu beiden senkrechte Wanderungsgeschwindigkeit abschätzen, die noch meßbar sein sollte.

So wurde dann Goldamalgam mit 0,1 Gew. $\%$ Gold untersucht, dessen Überführungszahl im elektrischen Feld gemessen ist ${ }^{1,2}$. Die Zelle dazu besteht im wesentlichen aus zwei senkrecht zum Magnetfeld liegenden Glasplatten, zwischen denen sich das Amalgam befindet. An den Längsseiten wird das Amalgam durch zwei Elektroden von $25 \mu$ Dicke begrenzt. Eine Querseite wird durch Araldit abgeschlossen, während die andere mit dem Amalgamvorrat in Verbindung steht. Das Amalgamblatt hat die Abmessungen $10 \mathrm{~cm} \times 1 \mathrm{~cm} \times 25 \mu$. Die Glasplatten werden mit Wasser gekühlt.

Zum Versuch wurde die vakuumdichte Zelle ausgepumpt und das flüssige Amalgam mit Atmosphärendruck

1 K. E. Schwarz, Z. Phys. Chem. (A) 161, 231 [1932]

2 P. C. Mangelsdorf JR., Symposium, Pittsburg, April 1959.

\begin{tabular}{|c|c|c|c|}
\hline $\begin{array}{c}\text { Probe } \\
\text { Nr. }\end{array}$ & $\begin{array}{c}\text { Amalgam } \\
\text { in g }\end{array}$ & $\begin{array}{c}\text { Gold } \\
\text { in } \gamma\end{array}$ & $\begin{array}{c}\text { Goldkonzentration } \\
\text { in Gew.\% }\end{array}$ \\
\hline 0 & 0,2312 & 238 & 0,103 \\
1 & 0,5724 & 590 & 0,103 \\
2 & 0,0917 & 88 & 0,097 \\
3 & 0,0966 & 84 & 0,087 \\
4 & 0,1250 & 73 & 0,058 \\
5 & 0,1242 & 40 & 0,032 \\
\hline
\end{tabular}

Tab. 1 .

hineingepreßt. Sechs Tage lang flossen $15 \mathrm{~A}$ in einem Magnetfeld von 5500 Gauss. Die Wasserkühlung führte dabei die im Amalgam erzeugte Wärme ab. Danach wurde der Strom langsam heruntergeregelt und dann erst das Magnetfeld abgeschaltet. Beim Eintauchen der Zelle in ein Kühlbad erstarrte das Amalgam, so daß die beiden Glasplatten mit dem Amalgam in etwa 2,5 cm breite Streifen zerbrochen werden konnten. Nach dem Wiegen des Amalgams wurde das Quecksilber abgetrieben und das zurückbleibende Gold mit Königswasser aufgelöst und als Kolloid kolorimetrisch bestimmt ${ }^{3}$.

Die Tabelle gibt die Analysenergebnisse wieder. Die ersten beiden Proben sind aus dem Amalgamvorrat entnommen. Die Proben zwei bis fünf sind die Abschnitte des Amalgamblattes. Neben diesem Versuch mit Platin-

3 E. B. SAndell, Colorimetric Determination of Traces of Metals, Interscience, Publ., Inc., New York 1950, 2nd Edition. 
elektroden wurde ein solcher mit Tantalelektroden durchgeführt. Er lieferte das gleiche Ergebnis.

Es ist bekannt, daß senkrecht zu dem elektrischen und zu dem magnetischen Feld in flüssigen Metallen ein Druckgradient auftritt. Für den Massentransport zeigt die Analyse, daß das Gold entgegen der Richtung des Druckanstieges wandert. Da der Versuch in der Zeit beendet wurde, in der der Gleichgewichtszustand durch Rückdiffusion noch nicht eingetreten war, läßt sich aus den Meßwerten bei $600 \mathrm{~A} / \mathrm{cm}^{2}$ und $5500 \mathrm{Gau} \beta$ eine Wanderungsgeschwindigkeit von $2,2 \cdot 10^{-2} \mathrm{~cm} / \mathrm{h}$ entnehmen. Eine eventuell auftretende Vermischung durch Konvektion ist bei $25 \mu$ Plattenabstand gering. Die Wanderungsgeschwindigkeit wäre bei $20000 \mathrm{Gauß}$ und
$1 \mathrm{~V} / \mathrm{cm}$ etwa so groß, wie die anfangs erwähnte im elektrischen Feld bei $1 \mathrm{~V} / \mathrm{cm}$.

In einer ausführlichen Veröffentlichung sollen demnächst die Experimente näher beschrieben und die Ergebnisse gedeutet werden.

Herrn Prof. A. Klemm danke ich für die Bereitstellung von Hilfsmitteln und für seine kritischen Diskussionsbemerkungen, Herrn Prof. J. Matтauch für die Erlaubnis, einen Magneten der massenspektrometrischen Abteilung des Institutes zu benutzen, und dem Bu n desministerium für Atomkernenergie und Wasserwirtschaft für finanzielle Unterstützung.

\section{Ein Beitrag der Versetzungen zur Anfangs- suszeptibilität in weichmagnetischem Material}

\author{
Von Georg Rieder
}

Institut für Technische Mechanik der TH Braunschweig

(Z. Naturforschg. 15 a, 746-748 [1960]; eingegangen am 24. Juni 1960)

Die Mechanismen, welche die Ummagnetisierungsvorgänge in schwachen Feldern und magnetisch weichem Material bestimmen, sind in ihren Einzelheiten noch keineswegs ausreichend geklärt. Einen sehr wesentlichen Beitrag bildet die Wandwölbungstheorie von Kersten ${ }^{1}$. Der nach dieser Theorie berechnete Temperaturverlauf der Anfangssuszeptibilität gibt in vielen Fällen die Meßergebnisse überraschend gut wieder ${ }^{1}$; allerdings ist nach Dietze $^{2}$ die Gültigkeit der Kerstenschen Überlegungen von einer sehr scharfen Bündelung der linienförmigen Hindernisse in der Richtung leichter Wandwölbung abhängig; immerhin lassen die neueren Ergebnisse der Theorie der Kristallplastizität in Verbindung mit Einzelheiten des gemessenen Temperaturverlaufs der Koerzitivkraft in Nickel-Einkristallen in manchen Fällen eine gewisse Bevorzugung solcher Richtungen vermuten ${ }^{3}$.

Die Einzelheiten der Wechselwirkung zwischen der B Lochschen Wand und den Hindernissen gehen in die Wandwölbungstheorie nicht ein; die Wanddicke wird vernachlässigt und bis zum Abreißen unveränderliches Festhalten an den Haftlinien angenommen. In Wahrheit wird sich die Wand entsprechend ihrer endlichen Dicke auch an der Haftstelle selbst etwas über dem Hindernis, z. B. einer Versetzung, verschieben. Der Beitrag dieses Effektes zur Anfangssuszeptibilität soll nun an einem besonders einfachen Beispiel überschlägig berechnet und mit dem Beitrag der Wandwölbung verglichen werden.

Wie aus Tab. (5.2) a. a. O. ${ }^{4}$ durch Anwendung der

1 M. Kersten, Z. angew. Phys. 8, 313, 382, 496 [1956].

2 H.-D. Dietze, Techn. Mitt. Krupp 15, 169 [1957]; vgl. auch ibid. 15, 23 [1957] u. Z. Phys. 149, 276 [1957].

3 G. Rieder, Berichte der Arbeitsgemeinschaft Ferromagnetismus 1959 (Tagung Berlin), Stuttgart, Dr. Riederer-Verlag, im Druck.

4 G. Rieder, Abh. d. Braunschw. Wiss. Ges. 11, 20 [1959]; vgl. auch Z. Naturforschg. 14 a, 96 [1959].
Formel von Peach und Koenler zu entnehmen ist ${ }^{5}$, befindet sich eine Schraubenversetzung geeigneten Vorzeichens in [110]-Richtung in der Mitte einer $\left(180^{\circ}\right.$, [112]) -Wand in Nickel im stabilen Gleichgewicht. Vernachlässigt man die durch das Spannungsfeld der Versetzung bedingten Störungen in der Struktur der BLochschen Wände, so kann man - bis auf den bei Lilley ${ }^{6}$ nicht völlig korrekt eingesetzten Beitrag der Magnetostriktion ${ }^{4}-$ mit Hilfe der von $\operatorname{Lilley}^{6}$ angegebenen Formeln den Verlauf der Kraft zwischen Versetzung und BLochscher Wand als Funktion des Abstandes $z$ von der Wandmitte angeben.

Sind nun $b$ der Burgers-Vektor (vgl. z. B. KRöNer ${ }^{7}$ ), $c_{66}^{* \prime \prime}$ eine reduzierte Elastizitätskonstante ${ }^{4}, \lambda_{100}$ und $\lambda_{111}$ die Magnetostriktionskonstanten und $\Phi^{\prime}$ der Azimutwinkel des Magnetisierungsvektors, so ist die Kraft je Längeneinheit der Versetzungslinie ${ }^{4,5}$

$$
p=\frac{b}{2} c_{66}^{* \prime \prime}\left(2 \lambda_{100}+\lambda_{111}\right) \sin 2 \Phi^{\prime} .
$$

Vernachlässigt man den Beitrag der Magnetostriktion, so ist nach LiLley

$$
\frac{\mathrm{d} z}{\mathrm{~d} \Phi^{\prime}}=\frac{2 \sqrt{3} \delta_{0}}{\sin ^{2} \Phi^{\prime} \sqrt{1+8 \operatorname{cotg}{ }^{2} \Phi^{\prime}}},
$$

wobei die reduzierte Einheit der Wandweite

$$
\delta_{0}=\sqrt{\frac{1}{2} C /|K|}
$$

nach Lilley aus der Austauschsteifigkeit $C^{8}$ und der Kristallenergiekonstanten $K$ zu berechnen ist. Ist dann $J_{\mathrm{s}}$ die Sättigungsmagnetisierung, $L$ die Dicke der Werssschen Bezirke und $s$ der Abstand der Versetzungslinien in der Blochschen Wand, so ist die reversible Suszeptibilität unter der - im gewählten Beispiel gut zutreffen-

5 G. Rieder, Vortrag bei der Jahrestagung der GAMM in Freiberg 1960; erscheint in der Z. angew. Math. Mech.

6 B. A. Lilley, Phil. Mag. (7) 41, 792 [1950].

7 E. Kröner, Kontinuumstheorie der Versetzungen und Eigenspannungen. Springer-Verlag, Berlin 1958.

8 W. F. Brown JR., J. Appl. Phys. 30, 62 S [1959]. 